\title{
Cosmic-ray-driven dynamo in galactic disks
}

\section{A parameter study}

\author{
M. Hanasz ${ }^{1}$, K. Otmianowska-Mazur² ${ }^{2}$ G. Kowal ${ }^{2,3}$, and H. Lesch ${ }^{4}$ \\ 1 Toruń Centre for Astronomy, Nicolaus Copernicus University, 87-148 Toruń/Piwnice, Poland \\ e-mail: mhanasz@astri.uni.torun.pl \\ 2 Astronomical Observatory, Jagiellonian University, ul. Orla 171, 30-244 Kraków, Poland \\ 3 Department of Physics and Astronomy, McMaster University, 1280 Main St. W., Hamilton, ON L8S 4M1, Canada \\ 4 Astronomical Observatory, Munich University, Scheinerstr. 1, 81679 Munich, Germany
}

Received 28 May 2008 / Accepted 19 December 2008

ABSTRACT

\begin{abstract}
Aims. We present a parameter study of the magnetohydrodynamical-dynamo driven by cosmic rays in the interstellar medium (ISM), focusing on the efficiency of magnetic-field amplification and the issue of energy equipartition between magnetic, kinetic, and cosmicray $(\mathrm{CR})$ energies.

Methods. We perform numerical CR-MHD simulations of the ISM using an extended version of ZEUS-3D code in the shearingbox approximation and taking into account the presence of Ohmic resistivity, tidal forces, and vertical disk gravity. CRs are supplied in randomly-distributed supernova ( $\mathrm{SN}$ ) remnants and are described by the diffusion-advection equation, which incorporates an anisotropic diffusion tensor.

Results. The azimuthal magnetic flux and total magnetic energy are amplified in the majority of models depending on a particular choice of model parameters. We find that the most favorable conditions for magnetic-field amplification correspond to magnetic diffusivity of the order of $3 \times 10^{25} \mathrm{~cm}^{2} \mathrm{~s}^{-1}$, SN rates close to those observed in the Milky Way, periodic SN activity corresponding to spiral arms, and highly anisotropic and field-aligned CR diffusion. The rate of magnetic-field amplification is relatively insensitive to the magnitude of SN rates spanning a range of $10 \%$ to $100 \%$ of realistic values. The timescale of magnetic-field amplification in the most favorable conditions is $150 \mathrm{Myr}$, at a galactocentric radius equal to $5 \mathrm{kpc}$, which is close to the timescale of galactic rotation. The final magnetic-field energies reached in the efficient amplification cases fluctuate near equipartition with the gas kinetic energy. In all models CR energy exceeds the equipartition values by a least an order of magnitude, in contrast to the commonly expected equipartition. We suggest that the excess of cosmic rays in numerical models can be attributed to the fact that the shearing box does not permit cosmic rays to leave the system along the horizontal magnetic field, as may be the case for true galaxies.
\end{abstract}

Key words. galaxies: ISM - galaxies: magnetic fields - magnetohydrodynamics (MHD) - ISM: cosmic rays -

ISM: kinematics and dynamics - ISM: magnetic fields

\section{Introduction}

An attractive idea of fast galactic dynamo was proposed by Parker (1992). The idea relies on two ingredients: (1) cosmic rays $(\mathrm{CR})$ continuously supplied to the disk by supernova ( $\mathrm{SN}$ ) remnants and (2) fast magnetic reconnection, which operates in current sheets and enables the dissipation and relaxation of the random magnetic-field components at the limit of vanishing resistivity. In the past decade, we have investigated the different elements, physical properties, and consequences of Parker's idea and scenario by means of analytical calculations and numerical simulations (Hanasz \& Lesch 1993, 1997, 1998, 2000, 2001, 2003a,b; Hanasz et al. 2002, 2004, 2006; Lesch \& Hanasz 2003; Otmianowska-Mazur 2003; Otmianowska-Mazur et al. 2007; Kowal et al. 2003, 2006)

The first complete 3D numerical model of the CR-driven dynamo was developed by Hanasz et al. (2004, 2006). In this paper, we perform a parameter study of the CR-driven dynamo model by examining the dependence of magnetic-field amplification on magnetic diffusivity, supernova rate determining the $\mathrm{CR}$ injection rate, temporal modulation of $\mathrm{SN}$ activity, grid resolution, and $\mathrm{CR}$ diffusion coefficients.

The principle of action of the CR-driven dynamo is based on the cosmic-ray energy supplied continuously by $\mathrm{SN}$ remnants.
Due to the anisotropic diffusion of cosmic rays and the horizontal magnetic-field configuration, cosmic rays tend to accumulate within the disc volume. However, the configuration stratified by vertical gravity is unstable with respect to the Parker instability. Buoyancy effects induce vertical and horizontal motions of the fluid and formation of undulated patterns, such as magnetic loops in frozen-in, predominantly-horizontal magneticfields. The presence of rotation in galactic disks implies a coherent twisting of the loops by means of the Coriolis force, which leads to the generation of small-scale, radial magneticfield components. The next phase is merging of small-scale loops by the magnetic-reconnection process to form large-scale, radial magnetic-fields. Finally, the differential rotation stretches the radial magnetic field to amplify the large-scale azimuthal magnetic-field component. The coupling of amplification processes of radial and azimuthal magnetic-field components results in an exponential growth of the large-scale magnetic field. The timescale of magnetic-field amplification, resulting from the action of the CR-driven dynamo, was found (Hanasz et al. 2004, 2006) to be equal to $140-250 \mathrm{Myr}$, depending on the galactocentric radius, which is comparable to the galactic rotation period.

The CR-dynamo experiments reported in the aforementioned papers relied on the energy of CRs accelerated in SN remnants. Gressel et al. (2008b,a) reported a series of non-ideal 
MHD simulations demonstrating dynamo action resulting from the SN-driven turbulence, in the absence of CRs. Using a similar set of galactic disk parameters, with angular velocity a factor of 4 higher than the value of $0.025 \mathrm{Myr}^{-1}$, typical of the galactocentric radius of Sun, these authors found amplification of the large-scale magnetic fields on a timescale of $250 \mathrm{Myr}$. This indicates some similarity between the CR-driven dynamo and the dynamo driven by thermal energy output from supernovae. The similarity is presumably related to the buoyancy effect, which can be commonly attributed to the excess of both thermal and cosmic ray energies in the disk volume.

The magnitudes of galactic magnetic fields are usually estimated from measurements of the radio synchrotron-emission produced by cosmic ray electrons in the magnetic field. To interpret the radio emission spectrum, it is usually assumed that the energy density in the magnetic field is of the same order of magnitude as the energy density in cosmic-ray protons (which are assumed to outnumber the electrons by 100 to 1 , as they do in our Galaxy). There is however no compelling evidence of energy equipartition. Since the equipartition or minimum-energy assumption is one of the few ways of calculating radio source parameters, it is important to determine suitability of the approach. Strong et al. (2007) and Snodin et al. (2006) raised again the question about the applicability of the equipartition argument.

From the observational point of view (Fitt \& Alexander 1993; Vallee 1995), the equipartition assumption seems to hold. In particular, Vallee's comparison of three different methods determining galactic magnetic-field strengths (Faraday rotation method, equipartition method, and cosmic-ray equipartition) indicates that the equipartition fields are in a good agreement. On the other hand, Beck \& Krause (2005) considered in detail a problem raised by Chi \& Wolfendale (1993), which was that the commonly used classical equipartition or minimum-energy estimate of total magnetic-field strengths from radio-synchrotron intensities is of limited practical use because it is based on the hardly known ratio $\mathrm{K}$ of the total energies of cosmic-ray protons and electrons and also has inherent problems. They present a revised formula using the number-density ratio $\mathrm{K}$ for which they provided estimates. For particle acceleration in strong shocks, K is about 40 and increases with decreasing shock strength. Their revised estimate for the field strength inferred higher values than the classical estimate for flat radio spectra with spectral indices of about $0.5-0.6$, but smaller values for steep spectra and total fields stronger than about $10 \mu \mathrm{G}$. In young supernova remnants, for example, the classical estimate may be too high by up to 10 . On the other hand, if energy losses of cosmic-ray electrons are important, $\mathrm{K}$ increases with particle energy and the equipartition field may be underestimated significantly.

From a more global point-of-view the assumption of equipartition appears to be natural. A thermodynamical system will always distribute the free energy to all the degrees of freedom available, if the system has time to do so. In the case of accelerated particles diffusing in the large-scale and turbulent magnetic fields, one can expect that at least the turbulent magnetic field (since it represents three degrees of freedom) is virialized with respect to any other pressure term, such as cosmic-ray pressure. This may not be true for the ordered magnetic fields, which are supposed to be amplified by the combined action of differentially-rotating shear-flows in the disk and some helical upward and downward motion driven either by cosmic-ray pressure or any activity in the disk. In any case, the connection between star-formation activity accompanied by enhanced flux of cosmic rays and the amplification of large-scale magnetic fields, inherently raises the expectation that magnetic fields should not exhibit higher pressures than cosmic rays. One would expect to find instead magnetic fields whose pressure is lower than the pressure of the cosmic rays, if the cosmic rays represent a source of the galactic dynamo.

The paper is organized as follows: in Sect. 2, we describe the CR-driven dynamo-model and its numerical implementation; in Sect. 3, we present our simulation setup and describe parameters used in numerical simulations; and in Sect. 4, we describe results, focusing on the effect of each parameter on magneticfield amplification-rate. We discuss the final saturated states of models in terms of equipartition between kinetic, magnetic, and CR energies. Finally, in Sect. 5 we present our conclusions.

\section{Description of the model}

As in papers by Hanasz et al. (2004, 2006), we take into account the following elements of the CR-driven dynamo:

(1) the cosmic-ray component, a relativistic gas, which is described by the diffusion-advection transport equation (see Hanasz \& Lesch 2003b, for details of the numerical algorithm). The typical values of the diffusion coefficient found by modeling CR data (see e.g. Strong et al. 2007) are $(3-5) \times 10^{28} \mathrm{~cm}^{2} \mathrm{~s}^{-1}$ at energies $\sim 1 \mathrm{GeV}$, and even higher values of $10^{29} \mathrm{~cm}^{2} \mathrm{~s}^{-1}$ are possible (Jokipii 1999), although, we use lower values in a majority of our simulations;

(2) following Giacalone \& Jokipii (1999) and Jokipii (1999), we presume that cosmic rays diffuse anisotropically along magnetic-field lines. The ratio of the perpendicular to parallel CR diffusion coefficients propose by these authors was $5 \%$

(3) localized sources of cosmic rays: supernova remnants exploding randomly in the disk volume (see Hanasz et al. 2004). We assume that each SN remnant supplies cosmic rays almost instantaneously, i.e. the comic-ray input for a single SN remnant, distributed over several subsequent timesteps, equals $10 \%$ of the canonical SN kinetic-energy output $\left(=10^{51} \mathrm{erg}\right)$;

(4) resistivity of the ISM (see Hanasz et al. 2002; Hanasz \& Lesch 2003a; Tanuma et al. 2003) responsible for the onset of fast magnetic reconnection and topological evolution of magnetic-field lines. In this paper, we apply the uniform resistivity and neglect the Ohmic heating of gas by resistive dissipation of magnetic fields;

(5) shearing boundary conditions and tidal forces following the prescription by Hawley et al. (1995) aimed at modeling differentially rotating disks in the local, shearing-box approximation;

(6) realistic vertical disk gravity following the model of ISM in the Milky Way by Ferriere (1998).

The set of equations describing the model of the CR-driven dynamo includes resistive MHD and the cosmic-ray transport equations (see Hanasz et al. 2004):

$$
\begin{aligned}
& \frac{\partial \rho}{\partial t}+\boldsymbol{\nabla} \cdot(\rho \boldsymbol{V})=0, \\
& \frac{\partial e}{\partial t}+\boldsymbol{\nabla} \cdot(e \boldsymbol{V})=-p(\boldsymbol{\nabla} \cdot \boldsymbol{V}), \\
& \frac{\partial \boldsymbol{V}}{\partial t}+(\boldsymbol{V} \cdot \boldsymbol{\nabla}) \boldsymbol{V}=-\frac{1}{\rho} \boldsymbol{\nabla}\left(p+p_{\mathrm{cr}}+\frac{B^{2}}{8 \pi}\right) \\
& \quad+\frac{\boldsymbol{B} \cdot \boldsymbol{\nabla} \boldsymbol{B}}{4 \pi \rho}-2 \boldsymbol{\Omega} \times \boldsymbol{v}+2 q \Omega^{2} x \hat{\boldsymbol{e}}_{x}+g_{z}(z) \hat{\boldsymbol{e}}_{z},
\end{aligned}
$$


$\frac{\partial \boldsymbol{B}}{\partial t}=\boldsymbol{\nabla} \times(\boldsymbol{V} \times \boldsymbol{B})+\eta \Delta \boldsymbol{B}$,

$p=(\gamma-1) e, \quad \gamma=5 / 3$

where $q=-\mathrm{d} \ln \Omega / \mathrm{d} \ln R$ is the shearing parameter, $R$ is the distance to the galactic centre, $\eta$ is the resistivity, $\gamma$ is the adiabatic index of thermal gas, the gradient of cosmic ray pressure $\nabla p_{\mathrm{cr}}$ is included in the equation of motion (see e.g. Berezinskii et al. 1990), and other symbols have their usual meaning. The uniform resistivity is included only in the induction equation (see Hanasz et al. 2002). The thermal gas component is currently treated as an adiabatic medium.

The transport of the cosmic-ray component is described by the diffusion-advection equation (see e.g. Berezinskii et al. 1990; Schlickeiser \& Lerche 1985)

$\frac{\partial e_{\mathrm{cr}}}{\partial t}+\boldsymbol{\nabla}\left(e_{\mathrm{cr}} \boldsymbol{V}\right)=\boldsymbol{\nabla}\left(\hat{K} \boldsymbol{\nabla} e_{c r}\right)-p_{\mathrm{cr}}(\boldsymbol{\nabla} \cdot \boldsymbol{V})+Q_{\mathrm{SN}}$,

where $Q_{\mathrm{SN}}$ represents the source term for the cosmic-ray energydensity, and the rate of production of cosmic rays injected locally in the $\mathrm{SN}$ remnants is given by

$p_{\mathrm{cr}}=\left(\gamma_{\mathrm{cr}}-1\right) e_{\mathrm{cr}}, \quad \gamma_{\mathrm{cr}}=14 / 9$.

The adiabatic index of the cosmic-ray gas $\gamma_{\mathrm{cr}}$ and the formula for the diffusion tensor of

$K_{i j}=K_{\perp} \delta_{i j}+\left(K_{\|}-K_{\perp}\right) n_{i} n_{j}, \quad n_{i}=B_{i} / B$,

are adopted by following Ryu et al. (2003).

\section{Numerical simulations}

\subsection{Simulation setup}

In this paper we present a series of numerical simulations, whose aim was to search for the most favorable conditions for magnetic-field amplification by means of the CR-driven dynamo. The presented numerical simulations were performed with the aid of a Zeus-3D MHD code (Stone \& Norman 1992a,b) extended with additions to the standard algorithm, corresponding to items (1)-(6) of Sect. 2, i.e. the cosmic-ray component, treated as a fluid and described by the diffusion-advection equation, including anisotropic CR diffusion tensor and cosmic-ray sources - supernova remnants exploding randomly in the disk volume, resistivity of the ISM leading to magnetic reconnection, shearing-periodic boundary conditions, rotational pseudoforces, and a realistic vertical disk gravity.

All simulations were performed in a Cartesian domain of size $0.5 \mathrm{kpc} \times 1 \mathrm{kpc} \times 2 \mathrm{kpc}$ in $x, y$ and $z$ coordinates, corresponding to the radial, azimuthal, and vertical directions, respectively. The basic resolution of the numerical grid was $50 \times 100 \times 400$ grid cells in $x, y$, and $z$ directions, respectively, and for a smaller sample of simulations performed with higher values of $\mathrm{CR}$ diffusion coefficients the grid resolution was $25 \times 50 \times 200$ grid cells. The boundary conditions are shearedperiodic in coordinate $x$, periodic in coordinate $y$, and outflow on outer $z$-boundaries, with $e_{\mathrm{cr}}=0$ at the domain boundaries. The positions of $\mathrm{SN}$ are chosen randomly, with a uniform distribution in $x y$ coordinates and Gaussian distribution in $z$ coordinate.

The initial density distribution results from integration of the hydrostatic equilibrium equation for the vertical gravity model of Ferriere (1998) and the assumption of constant gas temperature across the disk, equal to approximately $6000 \mathrm{~K}$, which corresponds to a sound speed of about $7 \mathrm{~km} \mathrm{~s}^{-1}$. The integration procedure identifies hydrostatic equilibrium for a given gas column density treated as an input parameter.

The magnetic-field strength, incorporated in the initial hydrostatic equilibrium of gas, is defined by means of the parameter $\alpha$ by denoting the ratio of initial magnetic to gas pressures. The initial cosmic-ray pressure equals the initial gas pressure.

The CR energy supplied to the system in SN remnants, randomly distributed around the disk midplane, implies that the CR pressure-gradient force accelerates a vertical wind of thermal gas. To prevent significant mass losses from the computational domain, due to the vertical wind, we compensate the mass-loss $\Delta m$ after each timestep. The compensation mass is supplied as a mass source term, which is proportional to the initial mass distribution

$\Delta \rho(x, y, z)=\frac{\Delta m}{m_{\mathrm{tot}}} \rho_{0}(x, y, z)$,

where $m_{\text {tot }}$ is the total gas mass in the computational domain, and $\rho_{0}(x, y, z)$ is the initial density distribution, produced by the integration of the hydrostatic equilibrium equation.

\subsection{Simulation parameters}

The basic input parameters, resulting from the assumed model are the vertical gravity profile, local value of the galactic rotation and shear, gas column density, and supernova rate. We adopt these parameters from the global model of ISM in the Milky Way (Ferriere 1998) for the galactocentric radius $R=5 \mathrm{kpc}$, where angular velocity is $\Omega=0.05 \mathrm{Myr}^{-1}$, gas column density $\Sigma=27 \times 10^{20} \mathrm{~cm}^{-2}$, and the realistic vertical gravity given by Eq. (36) in (Ferriere 1998). In our simulations, the values of gas column density correspond to the total density of all gas components in Ferriere (1998), while SN-rate is the rate of type II supernovae. We assume for simplicity that all SN explosions appear as single supernovae, and that the vertical distribution of $\mathrm{SN}$ explosions is Gaussian of a fixed half-width equal to $100 \mathrm{pc}$.

In addition to the aforementioned well-established local disk-parameters, there is a group of less known quantities, such as effective magnetic diffusivity, CR diffusion coefficients, and efficiency of conversion of SN kinetic energy into cosmic ray energy. We assume the standard $10 \%$ value of kinetic-to-CR energy-conversion efficiency, and that the magnetic diffusivity and CR diffusion coefficients vary across a wide range.

In this paper, we present the results of five simulation series A-E. The summary of all variable simulations parameters for the entire set of simulations is presented in Table 1.

In the simulation series A (runs A1-A5), we examine the effects of magnetic-diffusivity variations on magnetic-field amplification by applying $\eta$ in the range $0-10^{3} \mathrm{pc}^{2} \mathrm{Myr}^{-1}$ corresponding to $0-3 \times 10^{26} \mathrm{~cm}^{2} \mathrm{~s}^{-1}$ in CGS units. We define the magnetic Reynolds number $\mathrm{Rm}=L_{y}^{2} \Omega / \eta$ as in Gressel et al. (2008b,a), where $L_{y}=1000 \mathrm{pc}$ is the domain size in the $y$ direction. We assume a continuous and time-invariable supply of $\mathrm{CRs}$ in $\mathrm{SN}$ remnants. The simulation runs $\mathrm{A} 1, \mathrm{~A} 2$ and $\mathrm{A} 3$ are the same as the runs B, C, and D, respectively, discussed by Otmianowska-Mazur et al. (2007). For comparison, we note, that the commonly adopted value of turbulent diffusivity in the ISM is $\eta_{\text {turb }} \simeq 1 / 3 v_{\text {turb }} L_{\text {turb }} \sim 10^{26} \mathrm{~cm}^{2} \mathrm{~s}^{-1}\left(\simeq 1 / 3 \times 10^{3} \mathrm{pc}^{2} \mathrm{Myr}^{-1}\right)$ for $v_{\text {turb }}=10 \mathrm{kms}^{-1}$ and $L_{\text {turb }}=100 \mathrm{pc}$. We note that the adopted values of magnetic diffusivity exceed the value corresponding to the Spitzer resistivity $\left(\simeq 10^{8} \mathrm{~cm}^{2} \mathrm{~s}^{-1}\right.$; see Parker 1992) by 15-18 orders of magnitude. The relative smallness of the Spitzer resistivity implies that an anomalous resistivity, considered as a subscale phenomenon, must be invoked to explain dissipation 
Table 1. Parameters of simulations presented in this paper.

\begin{tabular}{|c|c|c|c|c|c|c|c|c|}
\hline Simulation & $\overline{\alpha \alpha}$ & $\begin{array}{c} \\
{\left[\mathrm{pc}^{2} \mathrm{Myr}^{-1}\right]}\end{array}$ & $\overline{\mathrm{Rm}}$ & $\begin{array}{c}f_{\mathrm{SN}} \\
{\left[\mathrm{kpc}^{-2} \mathrm{Myr}^{-1}\right]} \\
\end{array}$ & $\begin{array}{c}f_{\mathrm{SN}} \text { modul. } \\
(\mathrm{Y} / \mathrm{N})\end{array}$ & $\begin{array}{c}K_{\|} \\
{\left[\mathrm{pc}^{2} \mathrm{Myr}^{-1}\right]}\end{array}$ & $\begin{array}{c}K_{\perp} \\
{\left[\mathrm{pc}^{2} \mathrm{Myr}^{-1}\right]}\end{array}$ & $\overline{\bar{n} n_{x} \times n_{y} \times n_{z}}$ \\
\hline A1 & $10^{-4}$ & 0 & $\infty$ & 130 & $\mathrm{~N}$ & $1 \times 10^{4}$ & $1 \times 10^{3}$ & $100 \times 50 \times 400$ \\
\hline A2 & $10^{-4}$ & 1 & $5 \times 10^{4}$ & 130 & $\mathrm{~N}$ & $1 \times 10^{4}$ & $1 \times 10^{3}$ & $100 \times 50 \times 400$ \\
\hline A3 & $10^{-4}$ & 10 & $5 \times 10^{3}$ & 130 & $\mathrm{~N}$ & $1 \times 10^{4}$ & $1 \times 10^{3}$ & $100 \times 50 \times 400$ \\
\hline A4 & $10^{-4}$ & 100 & $5 \times 10^{2}$ & 130 & $\mathrm{~N}$ & $1 \times 10^{4}$ & $1 \times 10^{3}$ & $100 \times 50 \times 400$ \\
\hline A5 & $10^{-4}$ & 1000 & $5 \times 10^{1}$ & 130 & $\mathrm{~N}$ & $1 \times 10^{4}$ & $1 \times 10^{3}$ & $100 \times 50 \times 400$ \\
\hline B1 & $10^{-4}$ & 0 & $\infty$ & 130 & $\mathrm{Y}$ & $1 \times 10^{4}$ & $1 \times 10^{3}$ & $100 \times 50 \times 400$ \\
\hline B2 & $10^{-4}$ & 1 & $5 \times 10^{4}$ & 130 & Y & $1 \times 10^{4}$ & $1 \times 10^{3}$ & $100 \times 50 \times 400$ \\
\hline B3 & $10^{-4}$ & 10 & $5 \times 10^{3}$ & 130 & Y & $1 \times 10^{4}$ & $1 \times 10^{3}$ & $100 \times 50 \times 400$ \\
\hline B4 & $10^{-4}$ & 100 & $5 \times 10^{2}$ & 130 & Y & $1 \times 10^{4}$ & $1 \times 10^{3}$ & $100 \times 50 \times 400$ \\
\hline B5 & $10^{-4}$ & 1000 & $5 \times 10^{1}$ & 130 & Y & $1 \times 10^{4}$ & $1 \times 10^{3}$ & $100 \times 50 \times 400$ \\
\hline $\mathrm{C} 1$ & $10^{-4}$ & 100 & $5 \times 10^{2}$ & 15 & $\mathrm{Y}$ & $1 \times 10^{4}$ & $1 \times 10^{3}$ & $100 \times 50 \times 400$ \\
\hline $\mathrm{C} 2$ & $10^{-4}$ & 100 & $5 \times 10^{2}$ & 30 & Y & $1 \times 10^{4}$ & $1 \times 10^{3}$ & $100 \times 50 \times 400$ \\
\hline C3 & $10^{-4}$ & 100 & $5 \times 10^{2}$ & 60 & Y & $1 \times 10^{4}$ & $1 \times 10^{3}$ & $100 \times 50 \times 400$ \\
\hline $\mathrm{C} 4$ & $10^{-4}$ & 100 & $5 \times 10^{2}$ & 250 & Y & $1 \times 10^{4}$ & $1 \times 10^{3}$ & $100 \times 50 \times 400$ \\
\hline $\mathrm{C} 5$ & $10^{-4}$ & 100 & $5 \times 10^{2}$ & 500 & $\mathrm{Y}$ & $1 \times 10^{4}$ & $1 \times 10^{3}$ & $100 \times 50 \times 400$ \\
\hline D1 & $10^{-4}$ & 100 & $5 \times 10^{2}$ & 130 & $\mathrm{~N}$ & $1 \times 10^{4}$ & $1 \times 10^{3}$ & $50 \times 25 \times 200$ \\
\hline D2 & $10^{-4}$ & 100 & $5 \times 10^{2}$ & 130 & $\mathrm{Y}$ & $1 \times 10^{4}$ & $1 \times 10^{3}$ & $50 \times 25 \times 200$ \\
\hline E1 & $10^{-2}$ & 100 & $5 \times 10^{2}$ & 130 & $\mathrm{~N}$ & $1 \times 10^{4}$ & $3 \times 10^{3}$ & $50 \times 25 \times 200$ \\
\hline E2 & $10^{-2}$ & 100 & $5 \times 10^{2}$ & 130 & $\mathrm{~N}$ & $3 \times 10^{4}$ & $1 \times 10^{3}$ & $50 \times 25 \times 200$ \\
\hline E3 & $10^{-2}$ & 100 & $5 \times 10^{2}$ & 130 & $\mathrm{~N}$ & $3 \times 10^{4}$ & $3 \times 10^{3}$ & $50 \times 25 \times 200$ \\
\hline E4 & $10^{-2}$ & 100 & $5 \times 10^{2}$ & 130 & $\mathrm{~N}$ & $3 \times 10^{4}$ & $1 \times 10^{4}$ & $50 \times 25 \times 200$ \\
\hline E5 & $10^{-2}$ & 100 & $5 \times 10^{2}$ & 130 & $\mathrm{~N}$ & $1 \times 10^{5}$ & $1 \times 10^{3}$ & $50 \times 25 \times 200$ \\
\hline E6 & $10^{-2}$ & 100 & $5 \times 10^{2}$ & 130 & $\mathrm{~N}$ & $1 \times 10^{5}$ & $3 \times 10^{3}$ & $50 \times 25 \times 200$ \\
\hline E7 & $10^{-2}$ & 100 & $5 \times 10^{2}$ & 130 & $\mathrm{~N}$ & $1 \times 10^{5}$ & $1 \times 10^{4}$ & $50 \times 25 \times 200$ \\
\hline
\end{tabular}

Subsequent columns show: simulation name, initial ratio of magnetic to gas pressure $\alpha$, magnetic diffusivity $\eta$, magnetic Reynolds number Rm, surface frequency of SN explosions, presence of SN-rate modulation, parallel $K_{\|}$and perpendicular $K_{\perp} \mathrm{CR}$ diffusion coefficients, and grid resolution in $x, y$ and $z$ directions.

of the small-scale magnetic fluctuations in the ISM. Following Parker (1992), we assume that reconnection rates in the ISM are comparable to those predicted by the Petscheck's fast reconnection model, i.e. the magnetic cutting-speeds are of the order of $v_{\mathrm{A}} / \log (\mathrm{Rm})$ rather than $v_{\mathrm{A}} / \sqrt{\mathrm{Rm}}$ typical for the slow ParkerSweet reconnection model, where $\mathrm{Rm}$ is the Lundquist number or the magnetic Reynolds number.

In the simulation series B (runs B1-B5), we apply the same range of magnetic diffusivity values, but the CR supply is modulated in a way that mimics passages of subsequent spiral arms, regulating the star formation rate and subsequent SN-rate. The effect of spiral arms is modelled (see Hanasz et al. 2006) by supplying cosmic rays in intermittent periods of $25 \mathrm{Myr}$, for SN rate equal to a factor of 4 higher than the reference $f_{\mathrm{SN}}$, and followed by periods of $75 \mathrm{Myr}$ without any SN activity. The time-averaged supernova rate in this case equals to the reference $f_{\mathrm{SN}}$.

In the simulation series $\mathrm{C}$ (runs $\mathrm{C} 1-\mathrm{C} 5$ ), we apply a constant magnetic diffusivity $\eta=100 \mathrm{pc}^{2} \mathrm{Myr}^{-1}$ and vary the surface frequency of SN explosions in the range of $15-500 \mathrm{kpc}^{-2} \mathrm{Myr}^{-1}$, assuming modulated CR supply as in the simulation series B. In the simulation series D (runs D1-D2), we repeat the simulations A4 and B4 with a grid resolution that is a factor of two lower in each spatial direction.

Due to the CFL timestep limitation of the currently used explicit algorithm of CR diffusion, the applied values of CR diffusion coefficients are lower than realistic values. The timestep limitation, which ensures stability of explicit numerical schemes applied to the diffusion equation, is $\Delta t \leq 0.5(\Delta x)^{2} / K$, where $K$ is the diffusion coefficient. The timestep becomes prohibitively short when the diffusion coefficient is large or the spatial step is too small. For this reason, the CR diffusion coefficient was reduced in simulation series A-D, by about one order of magnitude, with respect to the aforementioned realistic values $3-6 \times 10^{28} \mathrm{~cm}^{2} \mathrm{~s}^{-1} \simeq 1-2 \times 10^{5} \mathrm{pc}^{2} \mathrm{Myr}^{-1}$. The fiducial values of the parallel and perpendicular diffusion coefficients applied in simulation series A-D are, respectively, $K_{\|}=1 \times$ $10^{4} \mathrm{pc}^{2} \mathrm{Myr}^{-1} \simeq 3 \times 10^{27} \mathrm{~cm}^{2} \mathrm{~s}^{-1}$, and $K_{\perp}=1 \times 10^{3} \simeq$ $3 \times 10^{26} \mathrm{~cm}^{2} \mathrm{~s}^{-1}$.

Finally, in the simulation series E (runs E1-E6) we increase the parallel $K_{\|}$and perpendicular $K_{\perp}$ CR diffusion coefficients by factors 3 and 10, with respect to the fiducial values, to examine the magnetic-field amplification for more realistic magnitudes of these quantities. In this way, we apply realistic CR diffusion coefficients in a few single simulation runs, even though the maximum CR diffusion-coefficients used do not reach the upper range of realistic values, of the order of $10^{29} \mathrm{~cm}^{2} \mathrm{~s}^{-1}$, mentioned in the literature.

\section{Results}

In Fig. 1, we show the distribution of cosmic-ray gas and magnetic-field vectors (panel (a)), and thermal gas density and gas velocity vectors (panel (b)) in the $y z$-slice taken for $x=0$ at $t=1000 \mathrm{Myr}$.

We note in panel (a) that the dominating, horizontal, magnetic field component in the disk volume undulates in a way resembling the effects of Parker instability. The cosmic-ray energy-density is smoothed well by the diffusive transport in the computational volume. The vertical gradient of the cosmicray energy-density is maintained by the supply of cosmic rays around the equatorial plane of the disk in the presence of vertical gravity. The cosmic-ray energy-density is expressed in units in which the thermal, gas energy-density corresponds to $\rho=1 \mathrm{~cm}^{-3}$ and the isothermal sound speed $c_{\mathrm{s}}=7 \mathrm{~km} \mathrm{~s}^{-1}$ is 

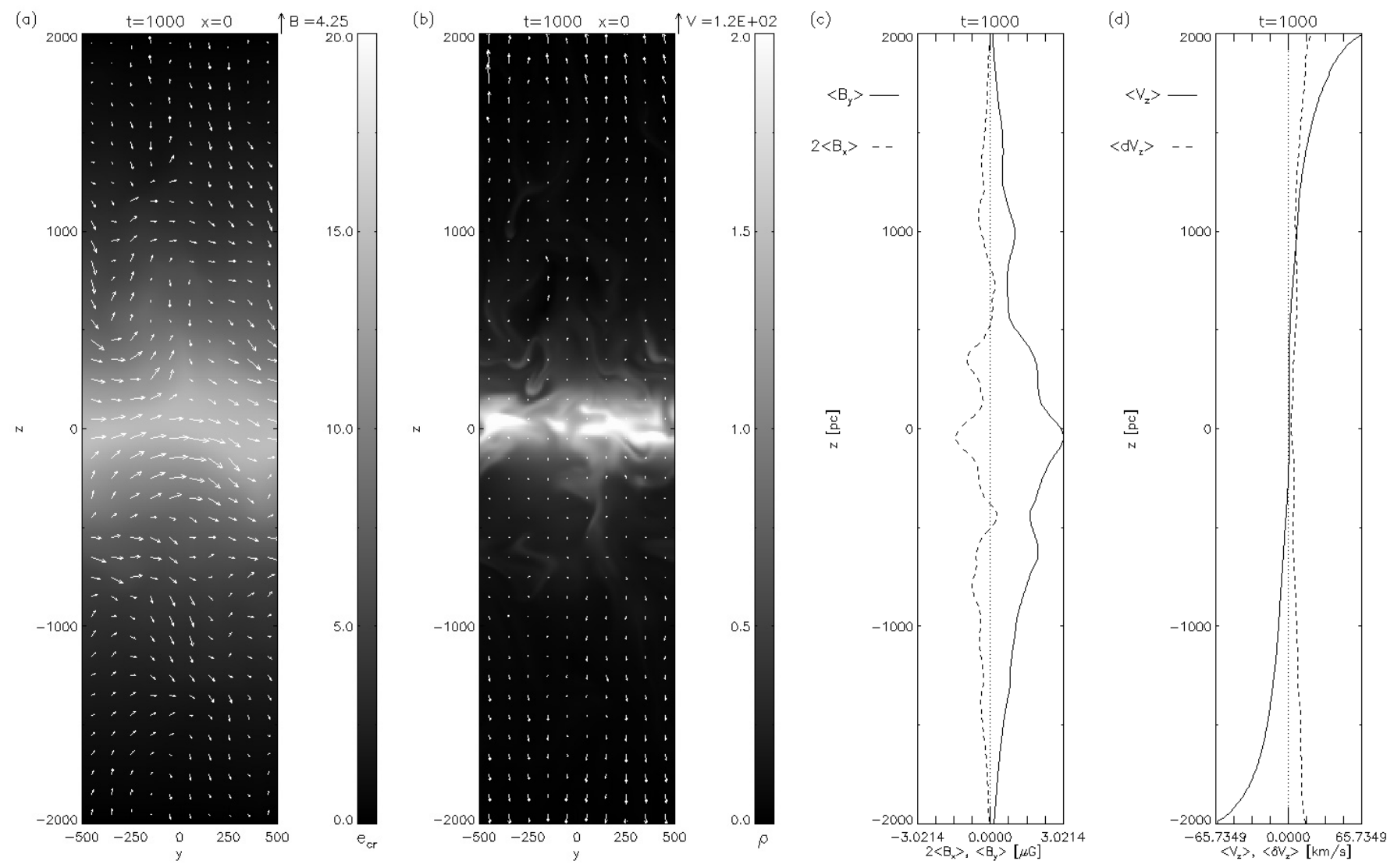

Fig. 1. Exemplary plots illustrating the state of the system at $t=1000 \mathrm{Myr}$ for simulation A4. In the first two panels we present slices through the computational volume in the $y z$-plane for $x=0$. Panel a) shows cosmic ray energy density with vectors of magnetic field, panel b) shows gas density with velocity vectors. In panel c) we plot horizontally averaged $x$ and $y$ components of magnetic field, and in panel d) horizontally averaged vertical velocity component and its fluctuations.

equal to 1 . The velocity field and the gas density are shown in panel (b). It is apparent that the distribution of gas is significantly less smooth that the distribution of cosmic rays.

To examine the structure of the large-scale field, we show in panel (c) the horizontally averaged magnetic field components $\left\langle B_{x}(z)\right\rangle$ and and $\left\langle B_{y}(z)\right\rangle$. A striking property of the mean magnetic-field configuration is the almost exact coincidence of peaks between the oppositely-directed radial and azimuthal field components. This feature resembles the standard picture of an $\alpha \omega$-dynamo: the azimuthal, mean magnetic-component is generated from the radial one and vice versa (see Lesch \& Hanasz 2003, for a correspondingly simple analytical model).

In panel (d) we show the horizontally-averaged vertical velocity-component $\left\langle V_{z}\right\rangle$ and its fluctuations $\left\langle\delta V_{z}\right\rangle$. It is apparent that the bulk speeds of the wind, driven by the vertical gradient of CR pressure, reach $65 \pm 15 \mathrm{~km} \mathrm{~s}^{-1}$ at $z=2 \mathrm{kpc}$. Vertical systematic winds of bulk speeds, comparable to rotational galactic velocities, influence large-scale structures of galactic magnetic fields and are observed in external starburst galaxies such as NGC 253 (see Heesen et al. 2007, 2009).

By varying the parameters discussed in the previous section, we intend to determine the regions of parameter space in which the magnetic-field amplification is the most efficient. The amplification of the regular magnetic-field is identified by the amplification of the total magnetic energy in the computational domain, associated with the amplification of the azimuthal magneticflux. The magnetic flux shown in subsequent plots represents an azimuthal flux averaged over all $x z$ slices (across $y$ direction) throughout the discretized computational-domain. In the subsequent subsections, we present the parameter study of the CR-driven dynamo, focusing on the efficiency of magnetic-field amplification and the issue of equipartition between magnetic, kinetic, and CR energies.

\subsection{Dependence of magnetic field amplification on magnetic diffusivity}

As a first step in our parameter study of the cosmic-ray-driven dynamo, we examine, in simulation series A, the effect of magnetic diffusivity on the efficiency of magnetic-field amplification. Time evolution of magnetic energy and magnetic flux are shown in Fig. 2. Magnetic flux plotted in the left panel of Fig. 2 is scaled in the following way. The initial magnetic-field induction is defined by the parameter $\alpha=p_{\text {mag }} / p_{\text {gas }}$, shown in the second column of Table. 1, where we apply Parker's convention to assign the inverse of plasma beta as $\alpha$. The adopted values of $\alpha$ are $10^{-4}$ and $10^{-2}$ in different simulations, while $\alpha=1$ implies that magnetic pressure equals the thermal gas pressure. We scale magnetic flux so that $\alpha=1$ corresponds to the azimuthal magnetic-flux $\Phi_{a}=1$. The total magnetic energy plotted in the right panel of Fig. 2 is scaled with respect to the timeaveraged total kinetic-energy in the computational domain. The latter quantity appears to fluctuate about a mean value, which is practically time-invariant for all simulation runs, thus this type of scaling is convenient. The scaling described above is applied to all subsequent plots of magnetic flux and magnetic energy.

The evolution of magnetic energy and magnetic flux in the models of simulation series A, represented by different 

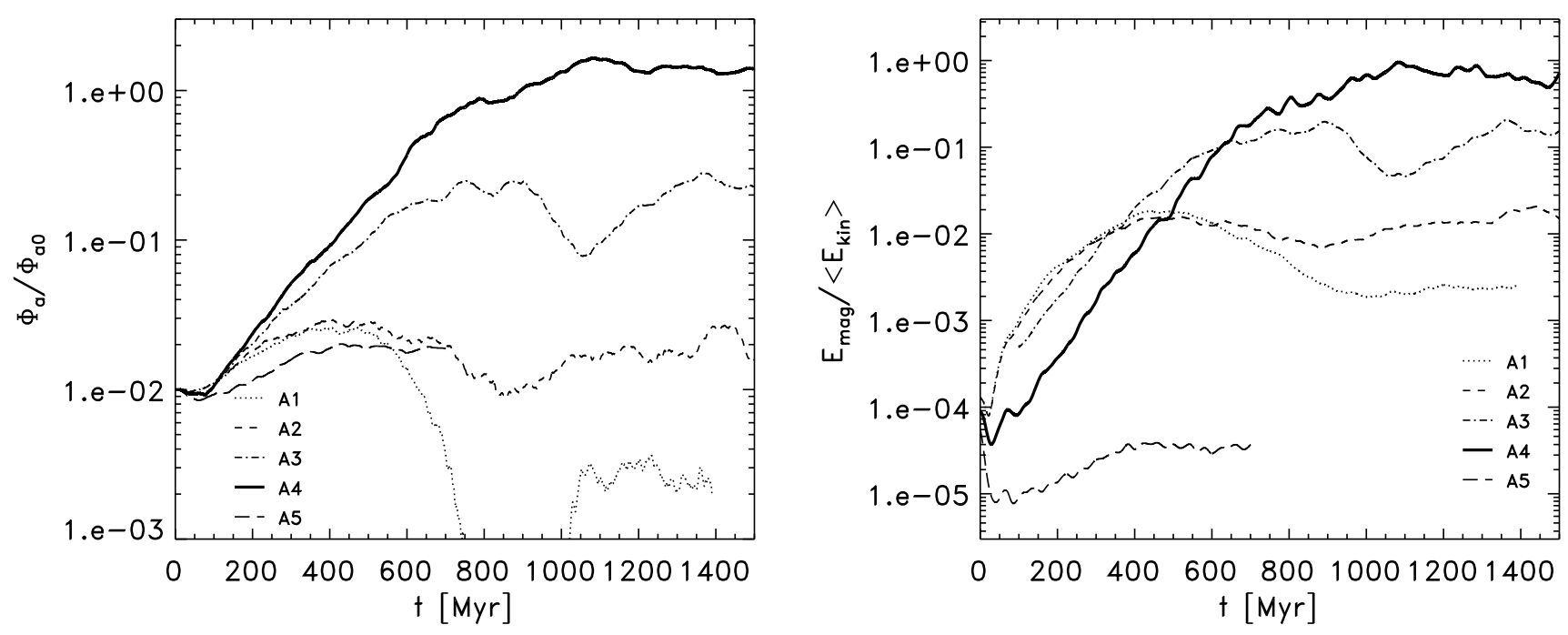

Fig. 2. Time evolution of azimuthal magnetic flux and total magnetic energy for different values of magnetic diffusivity in simulation series A. The curves represent respectively cases of $\eta=0$ (A1), $\eta=1$ (A2), $\eta=10$ (A3), $\eta=100$ (A4) and $\eta=1000$ (A5) in units $\mathrm{pc}^{2} \mathrm{Myr}^{-1}$.

curves in Fig. 2, illustrates that magnetic-field amplification depends strongly on magnetic diffusivity. In the case of vanishing explicit-resistivity, magnetic energy increases by about 2.5 orders of magnitude during the first $500 \mathrm{Myr}$, but magnetic flux is amplified by only a factor of 3 during this same period and decreases later on. This effect can be attributed to a predominant growth in the small-scale turbulent magnetic-field component with a little contribution of large-scale magnetic field amplification (see Otmianowska-Mazur et al. 2007, for a more extended analysis of the simulations presented in this section). We note, however, that numerical resistivity, always present in numerical MHD simulations, may influence the behavior of the simulation run $\mathrm{A} 1$, corresponding to $\eta=0$. The amount of numerical magnetic diffusivity $\eta \simeq 0.7 \mathrm{pc}^{2} \mathrm{Myr}^{-1}$ was quantified, for the present grid resolution, according to the Parker instability simulations by Kowal et al. (2003).

When magnetic diffusivity increases up to $\eta=$ $100 \mathrm{pc}^{2} \mathrm{Myr}^{-1}$, the efficiency of the magnetic-field amplification increases. For $\eta=100 \mathrm{pc}^{2} \mathrm{Myr}^{-1}$ (run A4), the growth in magnetic flux persists until $t=1000 \mathrm{Myr}$ and saturates in value thereafter. For smaller values of $\eta$, the growth rate is lower and the maximum values of magnetic flux are lower than those attained for $\eta=100 \mathrm{pc}^{2} \mathrm{Myr}^{-1}$. For negligible or small explicit diffusivity (runs A1 and A2), magnetic energy increases initially more rapidly than at higher resistivity (runs A3 and A4). This behavior means that low resistivity enables an initially more rapid growth of the random magnetic-field component, while for higher resistivity, random magnetic fields are quickly dissipated. The increase of the total magnetic energy follows closely, in the latter case, the increase in the mean magnetic flux. It is also apparent that the the amplification of the magnetic flux and magnetic energy for $\eta=1000 \mathrm{pc}^{2} \mathrm{Myr}^{-1}$ (run A5) is significantly lowered with respect to the other runs.

To explain the physical mechanism controlling magneticfield amplification by means of the magnitude of magnetic diffusivity, we plot the ratio of total (volume-integrated) energies of vertical to azimuthal magnetic-field components in Fig. 3. It is apparent that the energy in the vertical magnetic-field is higher than for all lower magnetic diffusivity runs A1-A3 ( $\eta=0,1$, and $10 \mathrm{pc}^{2} \mathrm{Myr}^{-1}$ ), and is comparable to the energy in the azimuthal

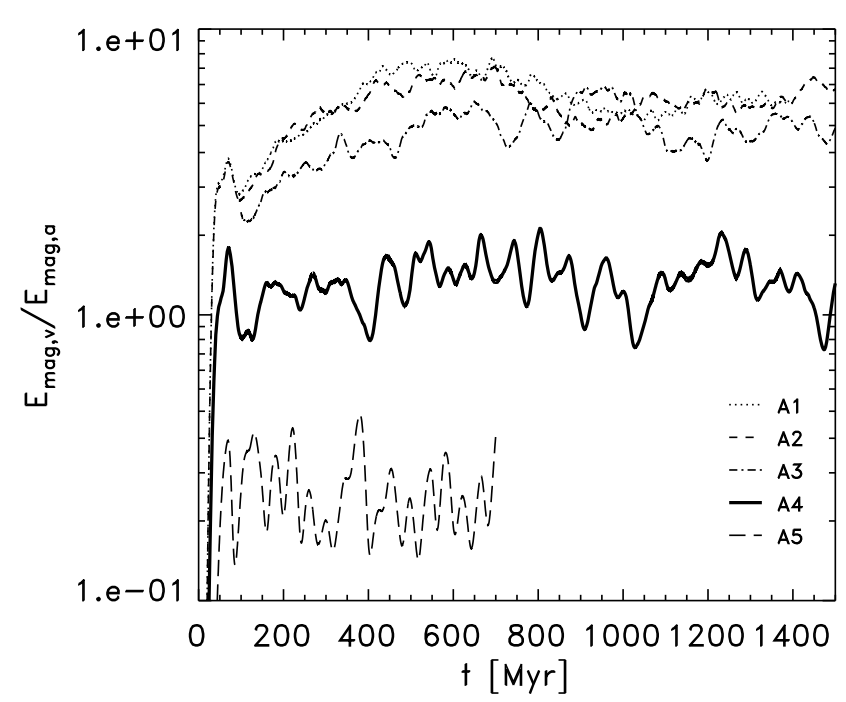

Fig. 3. Time evolution of the ratio of energies of vertical to horizontal magnetic-field components for different values of magnetic diffusivity in the simulation series A. Line assignments are the same as in Fig. 2.

magnetic-field of run A4 $\left(\eta=100 \mathrm{pc}^{2} \mathrm{Myr}^{-1}\right)$, providing the strongest magnetic-field amplification. Among all simulations in series A, only simulation A4 achieves energetic equipartition between the magnetic-field and gas kinetic energy as a result of the amplification process.

In the case of the high magnetic diffusivity (run A5, $\eta=$ $1000 \mathrm{pc}^{2} \mathrm{Myr}^{-1}$ ), energy in the vertical magnetic-field component, however, remains far lower than the energy in the azimuthal magnetic field, and magnetic-field amplification does not occur. This can be interpreted in terms of resistive damping of the undulatory mode of the Parker instability in favor of the interchange mode, which does not contribute to the dynamo action. The above finding indicates that the most favorable conditions for magnetic-field amplification correspond to approximately equal energies in the vertical and azimuthal magnetic fields in the case of buoyancy-driven dynamo. 
To demonstrate the effect of resistivity on kinetic and magnetic turbulent spectra, we compute the Fourier transforms of the kinetic and magnetic-energy densities, as in the paper by Otmianowska-Mazur et al. (2007). The results are shown in Fig. 4. The highly anisotropic nature of turbulence is reflected in the different lines representing Fourier transforms in the $x, y$ and $z$-directions. We find that the kinetic spectra, which generally emulate the Kolmogorov spectrum $\propto k^{-5 / 3}$, depend weakly on magnetic diffusivity. The high values of magnetic diffusivity $\eta=100-1000 \mathrm{pc}^{2} \mathrm{Myr}^{-1}$ lead only to the damping in the shortwavelength components of the Fourier spectrum computed in the $y$-direction.

The magnetic spectra appear to be far more sensitive to variations in magnetic diffusivity. The plots obtained for runs A1 and A2 exhibit practically identical spectra in all directions. This means that the diffusivity of $1 \mathrm{pc}^{2} \mathrm{Myr}^{-1}$ does not change the results for negligibly low explicit resistivity, or in other words the numerical resistivity of the code corresponds to the resistivity of Run A2. The effect of resistivity is evident in the smaller amplitude of the high- $k$ modes for $\eta=10 \mathrm{pc}^{2} \mathrm{Myr}^{-1}$ (Run A3) and an apparent cutoff the in magnetic spectra around $k / 2 \pi \simeq 0.02 \mathrm{pc}^{-1}$ for $\eta=100 \mathrm{pc}^{2} \mathrm{Myr}^{-1}$. A further increase in magnetic diffusivity up to $\eta=1000 \mathrm{pc}^{2} \mathrm{Myr}^{-1}$ leads to a significant reduction in amplitudes of all modes for the Fourier transforms performed in $z$-direction, steepening the entire spectrum in the $z$-direction and having a surprising effect on the flattening of the spectrum in the $x$-direction. The latter effect may indicate a qualitative change in the physical nature of the modes, which can plausibly attributed to the mentioned enhancement of the exchange mode of Parker instability.

We briefly note that we neglect any small-scale dynamics of the helical MHD turbulence. This assumption allows the uniformity of our diffusion coefficients, such as the magnetic diffusivity. Detailed investigations of the influence of small-scale helical MHD turbulence on galactic dynamos are presented by Maron \& Blackman (2002) and Maron et al. (2004).

We can interpret these results further in terms of the topological evolution of magnetic field, which is controlled by resistivity. The topology of magnetic field lines determines the paths of anisotropic cosmic-ray transport. For low values of resistivity, the buoyancy of the cosmic rays leads to an opening of magnetic-field lines toward the upper- and lower- $z$ boundaries. This implies that the diffusive escape of cosmic rays, along the open magnetic-field lines, dominates over the buoyancy and limits the effect of the Coriolis force, which is responsible for dynamo action.

\subsection{The effect of spiral arms}

In this section, we describe the simulation series $\mathrm{B}$ performed for the same set of simulation parameters as for series A with the exception that the cosmic-ray energy-input is currently modulated in time by a step function. Motivation for this type of CR supply is the presence of spiral arms in disk galaxies. We assume that $\mathrm{SNe}$ explode in arms at a rate that is proportional to the star formation rate. We assume that arms pass through the volume of our local computational domain once every $100 \mathrm{Myr}$, and that the arm passage takes $25 \mathrm{Myr}$, i.e. starting at $t=0$, we supply CRs for the first $25 \mathrm{Myr}$ of the $100 \mathrm{Myr}$ and cease the CR supply for the remaining $75 \mathrm{Myr}$. We enhance the $\mathrm{SN}$ rate in the spiral arms by a factor of 4 , so that the average $\mathrm{SN}$ rate during the entire period of density wave remains the same as in the simulation series A. The evolution in the mean magnetic flux and energy is presented in Fig. 5.
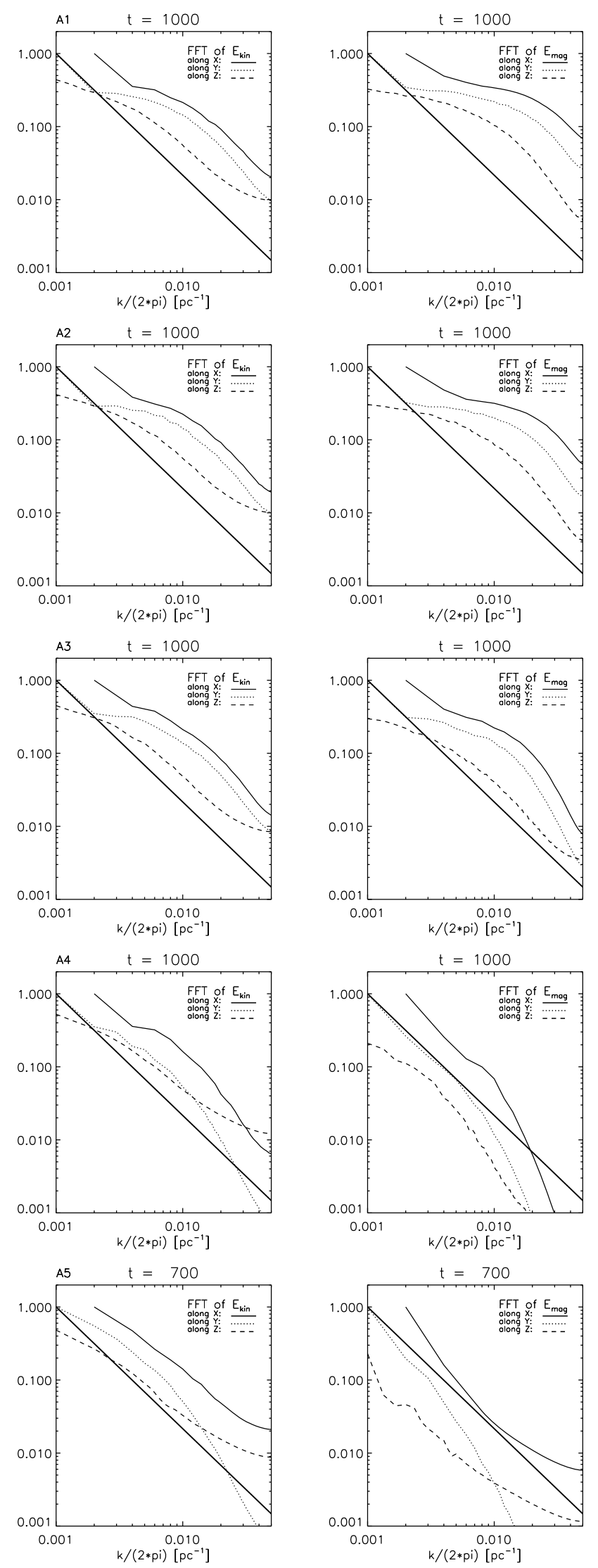

Fig. 4. Kinetic (left column) and magnetic (right column) spectra computed for $t=1000 \mathrm{Myr}$ (runs A1-A4) and $t=700 \mathrm{Myr}$ (Run A5), separately in $x, y$ and $z$-directions (full, dotted and dashed thin lines, respectively). Lines representing the $k^{-5 / 3}$ slope (thick full lines) are shown for comparison. 

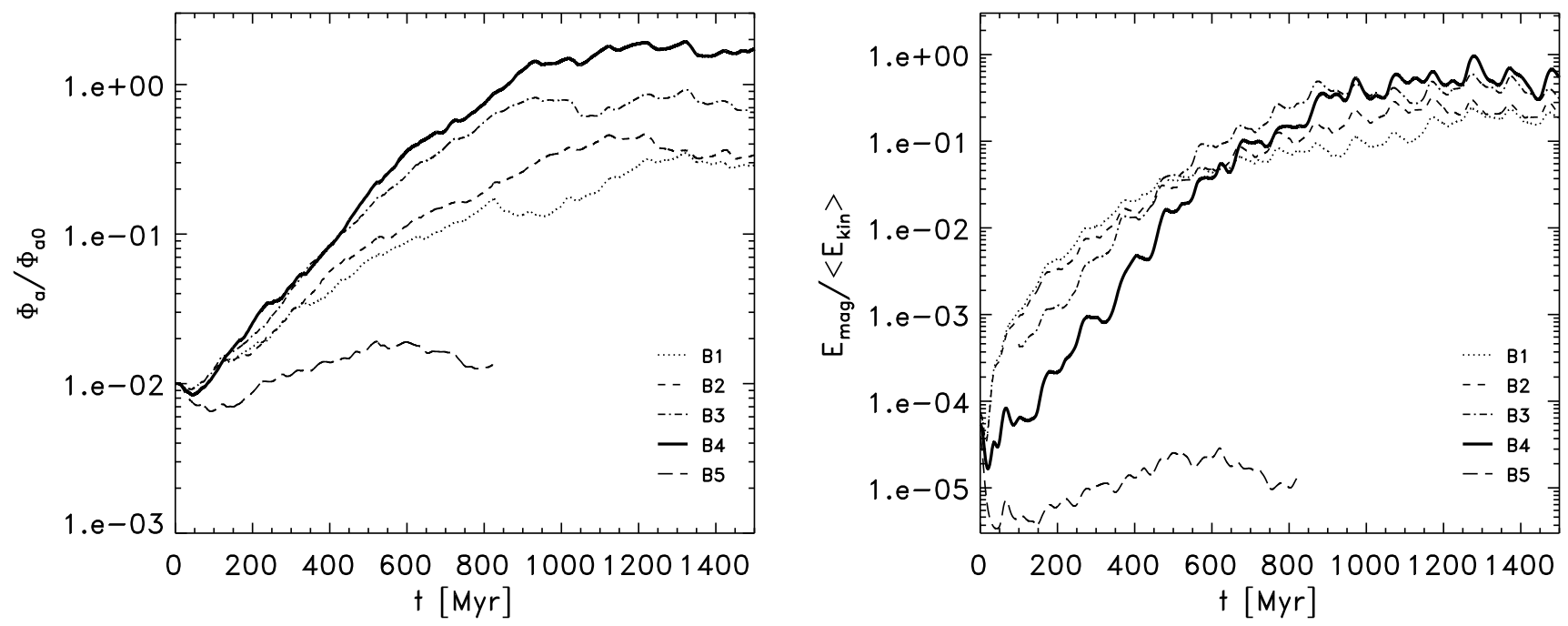

Fig. 5. Time evolution of azimuthal magnetic flux and total magnetic energy for different values of magnetic diffusivity, in presence of temporal modulations of SN-rate mimicking the presence of spiral arms in the simulation series B. The curves represent respectively cases of $\eta=0$ (B1), $\eta=1$ (B2), $\eta=10$ (B3), $\eta=100$ (B4) and $\eta=1000$ (B5) in units $\mathrm{pc}^{2} \mathrm{Myr}^{-1}$.

We find that in the present set of simulations, both magnetic flux and magnetic energy increase more rapidly than in the case of simulations without CR modulation. Magnetic-field amplification is now apparent even in simulations with $\eta=0$. The only exception is the $\eta=1000 \mathrm{pc}^{2} \mathrm{Myr}^{-1}$ simulation, which does not exhibit a noticeable amplification in the magnetic field, even in the presence of CR modulation. This indicates that the temporal modulation of $\mathrm{SN}$ rate acts in the same way as increasing magnetic diffusivity in the range of $\eta=0-100 \mathrm{pc}^{2} \mathrm{Myr}^{-1}$.

To interpret the above results, we suggest the following scenario: CRs supplied to the system trigger Parker instability and leave the disk volume by means of combined buoyant and diffusive transport. Vertical magnetic loops form efficiently during the period of enhanced SN activity, but later on, in the absence of CR perturbations, the magnetic field tends to relax before the next spiral-arm passage. In the absence of CR forcing in the inter-arm regions, even a small resistivity is sufficient to relax magnetic-field structure to a horizontal more regular configuration, which suppresses excessive losses of CRs by diffusive transport. Thus, the efficiency of the magnetic-field amplification is enhanced.

\subsection{Dependence of magnetic field amplification on SN-rate}

In all runs of the simulation series $\mathrm{A}$ and $\mathrm{B}$, we adopted the fiducial value of $f_{\mathrm{SN}}=130 \mathrm{kpc}^{-2} \mathrm{Myr}^{-1}$, derived from the global model of Milky Way by Ferriere (1998) for a galactocentric radius $R_{\mathrm{G}}=5 \mathrm{kpc}$. In this section, we describe the simulation series $\mathrm{C}$ performed for different $\mathrm{SN}$ rates of $f_{\mathrm{SN}}=$ $15,30,60,250$, and $500 \mathrm{kpc}^{-2} \mathrm{Myr}^{-1}$, together with simulation $\mathrm{B} 4\left(f_{\mathrm{SN}}=130 \mathrm{kpc}^{-2} \mathrm{Myr}^{-1}\right)$, to examine the effect of SN rate on magnetic-field amplification. The results are shown in Fig. 6. In all cases, the $\mathrm{SN}$ input is modulated as described in Sect. 4.2.

We note that the magnetic-field amplification rates and the final saturation levels of both magnetic flux and magnetic energy increase with $f_{\mathrm{SN}}$ as long as the $\mathrm{SN}$ rate is lower or equal to the fiducial, realistic value of $f_{\mathrm{SN}}=130 \mathrm{kpc}^{-2} \mathrm{Myr}^{-1}$ at a galactocentric radius $R_{\mathrm{G}}=5 \mathrm{kpc}$, when all other disk parameters are fixed. The e-folding times of magnetic flux deduced from the left panel of Fig. 6 are $150 \mathrm{Myr}$ for $f_{\mathrm{SN}}=130 \mathrm{kpc}^{-2} \mathrm{Myr}^{-1}$ (Run B4) and $190 \mathrm{Myr}$ for $f_{\mathrm{SN}}=15 \mathrm{kpc}^{-2} \mathrm{Myr}^{-1}$ (Run C1). We therefore note that the magnetic-field amplification rate is relatively insensitive to the magnitude of the SN rate for SN rates spanning one decade below the fiducial value. We also note that magnetic-field amplification saturates at the level of equipartition of magnetic and kinetic energies in the case of those simulation runs of series $\mathrm{C}$ for which the amplification holds.

When the SN rate is doubled, with respect to the fiducial value, then only a short period of magnetic-field amplification is observed, until $t=500 \mathrm{Myr}$, and if the SN rate is doubled once again then the magnetic field decays. The above results illustrate that magnetic-field amplification occurs for a wide range of $\mathrm{SN}$ rates, and that realistic values of $\mathrm{SN}$ rates correspond to those which are optimal for the galactic dynamo process. As in Sect. 4.1, we show the ratio of energies in the vertical to the horizontal magnetic-field components in Fig. 7. We find that for $\mathrm{SN}$ rates up to $f_{\mathrm{SN}}=130 \mathrm{kpc}^{-2} \mathrm{Myr}^{-1}$, the efficient magneticfield amplification is associated with the ratio of vertical to horizontal magnetic-field energies fluctuating about one, and, in the case of excessive CR supply $\left(f_{\mathrm{SN}}=250 \mathrm{kpc}^{-2} \mathrm{Myr}^{-1}\right.$ and more), the vertical magnetic-field energy dominates and the magnetic field ceases increasing in strength.

\subsection{Dependence of magnetic field amplification on the grid resolution}

To check the effect of the grid resolution on the simulation results, we increase the cell size to $(20 \mathrm{pc})^{3}$ in the simulations D1 and D2 and apply the same parameters as in simulations A4 and B4, respectively. In Fig. 8, we show the evolution in the total flux of the azimuthal magnetic field component and the total magnetic energy for simulations D1 and D2, and analogous curves for simulations A4 and B4, shown previously in Figs. 2 and 5. The results obtained at both resolutions are evidently similar, although a slightly more rapid increase in magnetic-field strength is observed in simulations performed at the lower resolution, which can be explained by the higher numerical resistivity. 

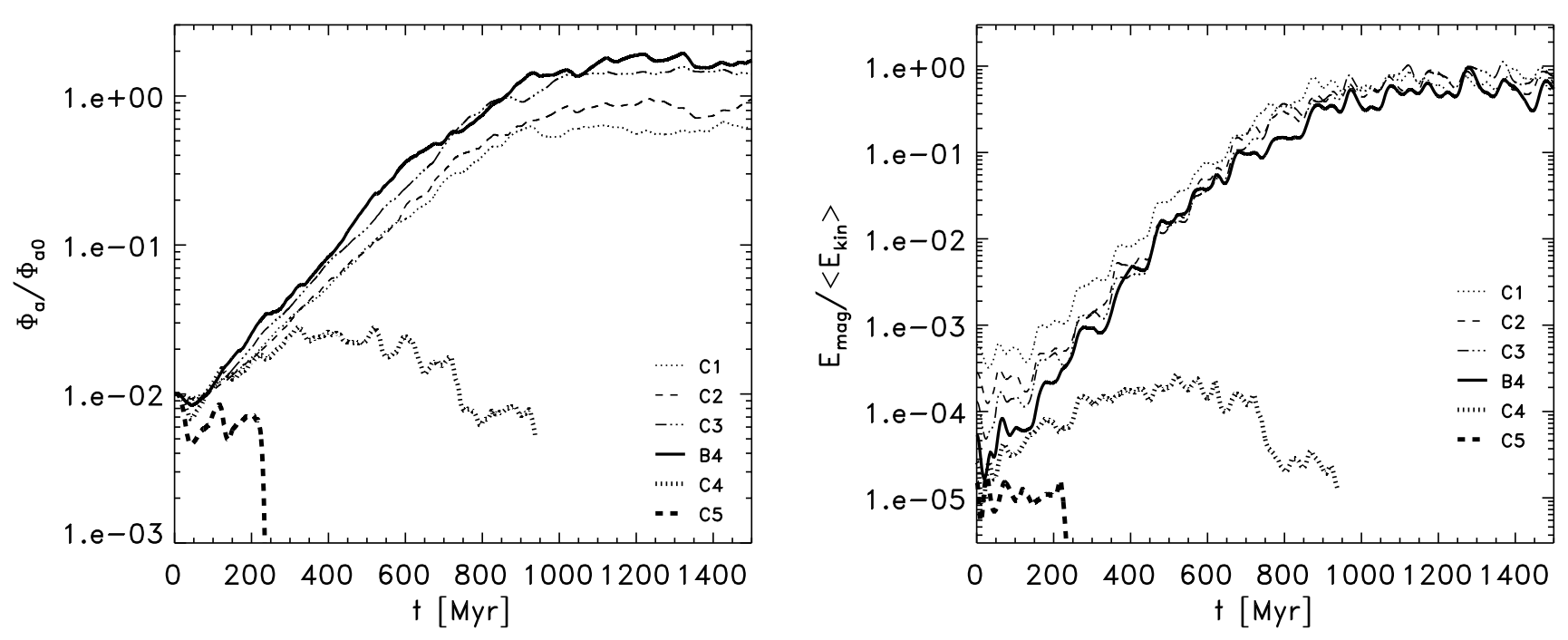

Fig. 6. Time evolution of azimuthal magnetic flux and total magnetic energy for different values of SN rate applied in simulation series $\mathrm{C}$, together with run B4, in the presence of temporal modulations of SN-rate. Line assignments are respectively: $f_{\mathrm{SN}}=15(\mathrm{C} 1), f_{\mathrm{SN}}=30(\mathrm{C} 2), f_{\mathrm{SN}}=60(\mathrm{C} 3)$, $f_{\mathrm{SN}}=130(\mathrm{~B} 4), f_{\mathrm{SN}}=250(\mathrm{C} 4)$, and $f_{\mathrm{SN}}=500(\mathrm{C} 5)$ supernova explosions per squared kpc per Myr.

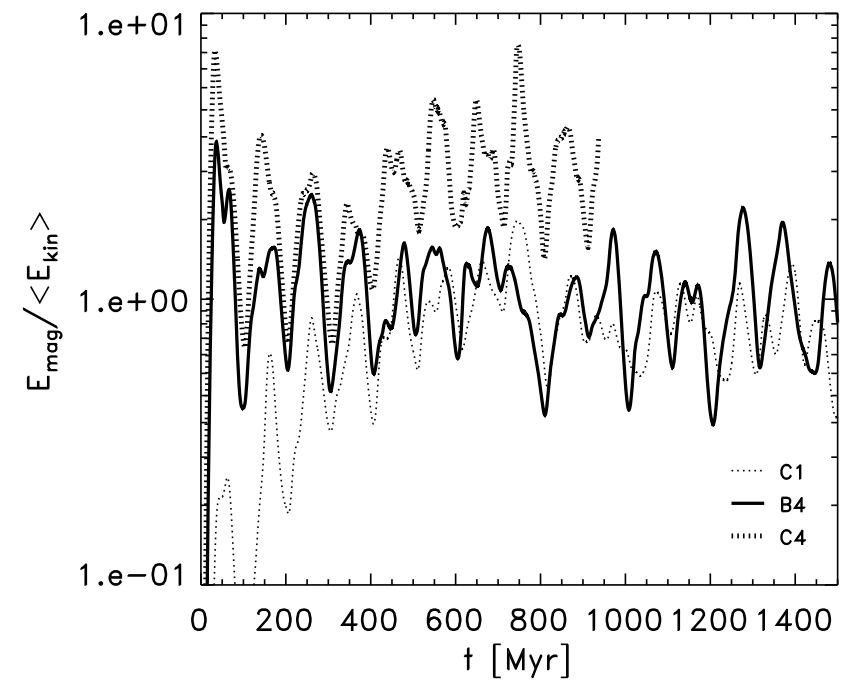

Fig. 7. Time evolution of the ratio of energies of vertical to horizontal magnetic field components for different (modulated) SN rates $f_{\mathrm{SN}}=15$ $(\mathrm{C} 1), f_{\mathrm{SN}}=130(\mathrm{~B} 4)$ and $f_{\mathrm{SN}}=250(\mathrm{C} 4)$ supernova explosions per squared kpc per Myr.

\subsection{Dependence of magnetic-field amplification on CR-diffusion coefficients}

The aim of simulation series $\mathrm{E}$ is to examine the effect of variations in the CR-diffusion coefficients on magnetic-field amplification. All simulations in series $\mathrm{E}$ are performed with resolution $(20 \mathrm{pc})^{3}$. This lower grid resolution makes it possible to enlarge the CR diffusion coefficients to realistic values, while preserving acceptable timesteps in the explicit integration algorithm of the $\mathrm{CR}$ diffusion-advection equation.

In the present simulation series $\mathrm{E}$, we vary both the parallel and perpendicular diffusion coefficients, choosing different pairs from the set of $K_{\|}=1 \times 10^{4}, 3 \times 10^{4}$ and $1 \times 10^{5} \mathrm{pc}^{2} \mathrm{Myr}^{-1}$, and $K_{\perp}=1 \times 10^{3}, 3 \times 10^{3}$ and $1 \times 10^{4} \mathrm{pc}^{2} \mathrm{Myr}^{-1}$. The results of these new simulations compared with those of simulation D1 are presented in Fig. 9.

The results of simulation series E can be summarized as follows. We note that the magnetic-field growth rate and the saturation values of magnetic flux and energy depend in particular on the choice of $K_{\|}$and $K_{\perp}$. When $K_{\|}$is increased by a factor of 3 and 10 with respect to D1, the initial growth in the magnetic field increases slightly, and the saturation level decreases by a factor of $2-3$, provided that $K_{\perp}$ is not too high. For $K_{\|}=3 \times 10^{4} \mathrm{pc}^{2} \mathrm{Myr}^{-1}$, the amplification holds for $K_{\perp}=$ $1 \times 10^{3} \mathrm{pc}^{2} \mathrm{Myr}^{-1}$ and $3 \times 10^{3} \mathrm{pc}^{2} \mathrm{Myr}^{-1}$, but for $K_{\perp}=1 \times 10^{4}$, the magnetic field decays. Similarly, for $K_{\|}=1 \times 10^{5} \mathrm{pc}^{2} \mathrm{Myr}^{-1}$, amplification holds for $K_{\perp}=1 \times 10^{3} \mathrm{pc}^{2} \mathrm{Myr}^{-1}$ and $K_{\perp}=$ $3 \times 10^{3} \mathrm{pc}^{2} \mathrm{Myr}^{-1}$, but for $K_{\perp}=1 \times 10^{4} \mathrm{pc}^{2} \mathrm{Myr}^{-1}$, we detect an initial growth only until $t=450 \mathrm{Myr}$ and decay thereafter. The present results indicate that magnetic-field amplification is possible only for $K_{\perp} \leq 3 \times 10^{3} \mathrm{pc}^{2} \mathrm{Myr}^{-1} \simeq 10^{27} \mathrm{~cm}^{2} s^{-1}$. Therefore, the anisotropy in the CR diffusion seems to be a crucial condition for magnetic-field amplification in the process of the CR-driven dynamo.

In the subsequent Fig. 10, we show the energy ratio of vertical to azimuthal magnetic-field components for simulations E4, E5 and E6, corresponding to three different values of $K_{\perp}$ and $K_{\|}=1 \times 10^{5} \mathrm{pc}^{2} \mathrm{Myr}^{-1}$. Comparing Figs. 9 and 10 we find that in the case of two simulation runs E4 and E5 (two lower values of $K_{\perp}$ ) the energy ratio of vertical to azimuthal magneticfield components varies in the range $\sim 0.3 \div 2$, corresponding to an efficient growth in magnetic energy. For the highest value of $K_{\perp}=10^{4}$, the magnetic-energy ratio increases occasionally by an order of magnitude, and the magnetic field decays.

\subsection{The issue of energy equipartition}

The results presented so far demonstrate that magnetic fields amplified by the CR-driven dynamo saturate close to the equipartition of magnetic and gas kinetic energies. It is commonly expected that CRs also remain in energetic equipartition with the gas and magnetic field. 

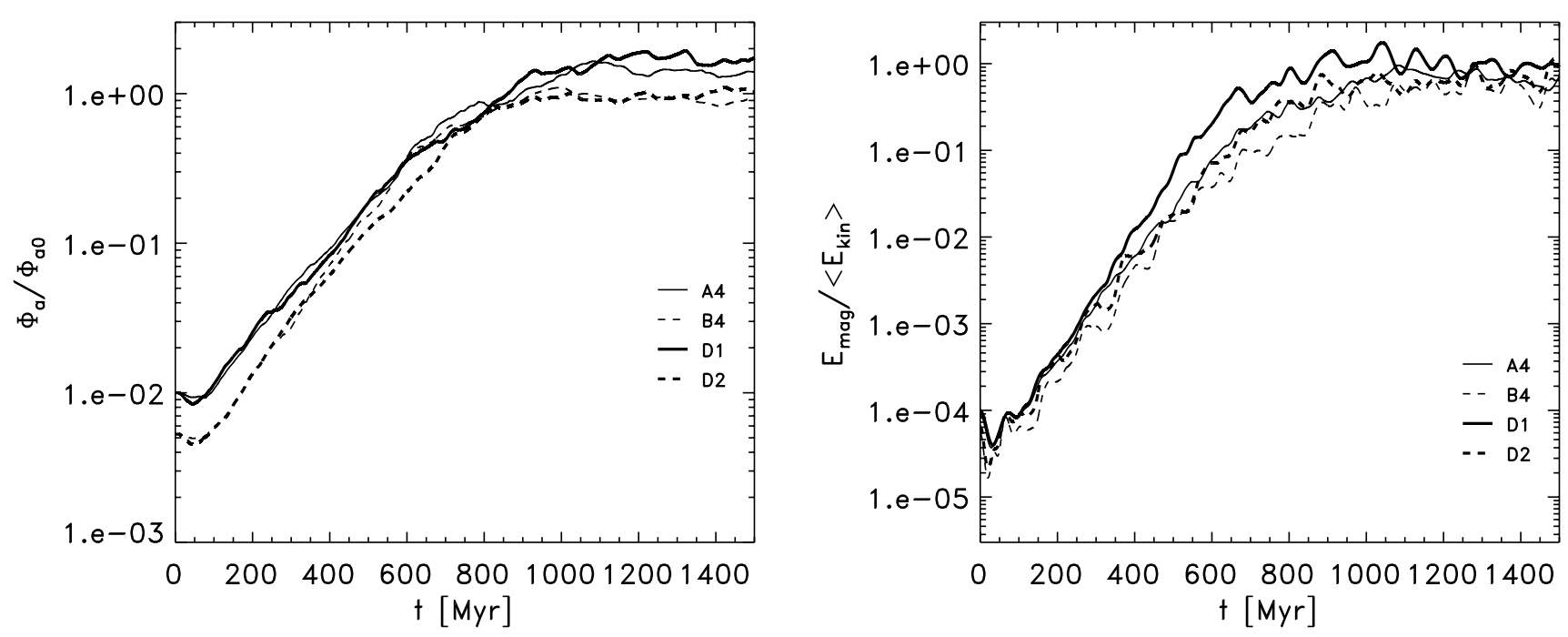

Fig. 8. Time evolution of the azimuthal magnetic flux and the total magnetic energy for simulations with grid resolutions (10 pc) ${ }^{3}$ (runs A4 and B4) and (20 pc) $)^{3}$ (runs D1 and D2).
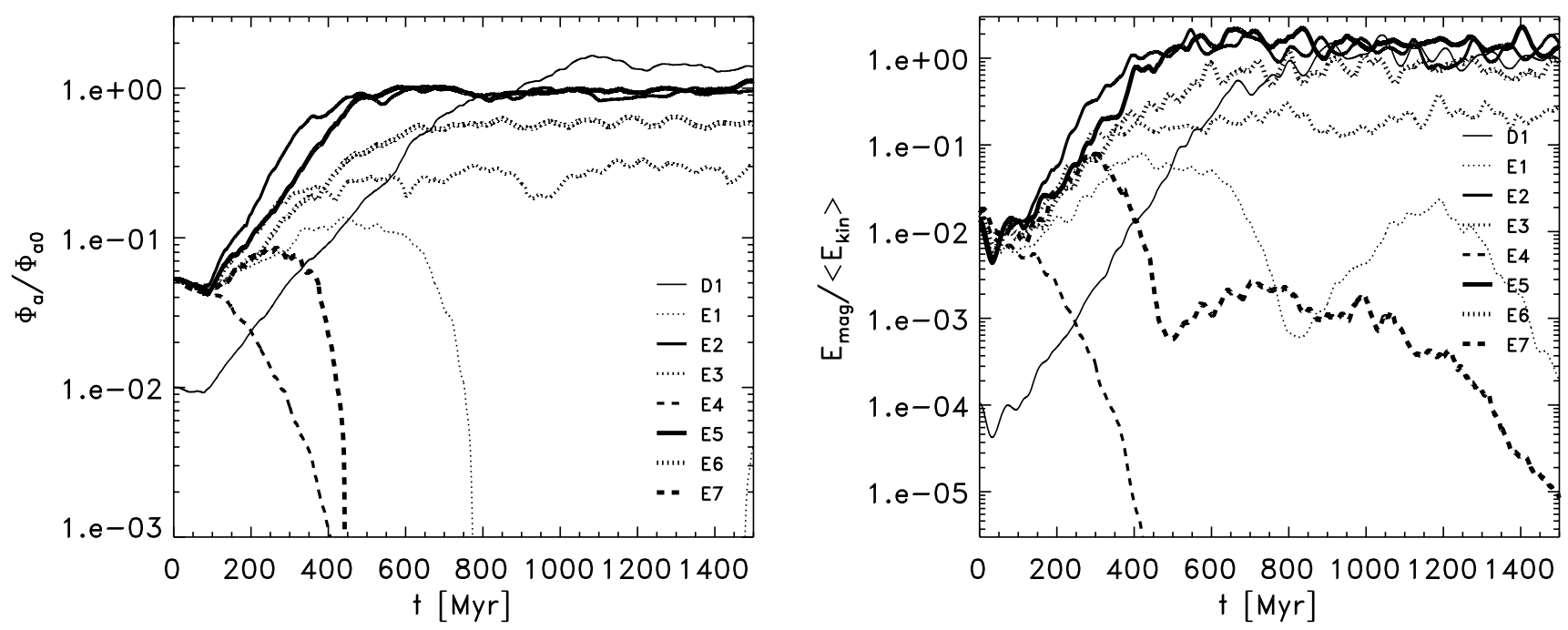

Fig. 9. Time evolution of the azimuthal magnetic flux and the total magnetic energy for different values of the parallel and perpendicular CR diffusion coefficients. Thin lines are used for $K_{\|}=1 \times 10^{4}$ (runs D1 and E1), mid lines are used for $K_{\|}=3 \times 10^{4}$ (runs E2 E3 and E4) and thick lines are used for $K_{\|}=1 \times 10^{5}$ (runs E5 E6 and E7). Full lines denote $K_{\perp}=1 \times 10^{3}$ (runs D1, E2 and E5), dotted lines denote $K_{\perp}=3 \times 10^{3}$ (runs E1, E3 and E6), dashed lines $K_{\perp}=1 \times 10^{4}$ (runs E4 and E7). All diffusion coefficients given in units $\mathrm{pc}^{2} \mathrm{Myr}^{-1}$.

To investigate the relationship between $\mathrm{CR}$ and other forms of energies, in Fig. 11 we plot the time evolution in the ratio of the CR to the time-averaged turbulent kinetic energy (after subtracting the kinetic energy of the large-scale shear flow) for different values of CR diffusion coefficients (the simulation series E). Depending on the diffusion coefficients, we find that, $\mathrm{CR}$ energy is higher than the turbulent kinetic energy by a factor of 10-50, while the magnetic-field energy, according to the results presented in Fig. 9, remains close to the gas turbulent energy.

For the first few hundred Myr, CRs appear to accumulate quickly in the disk since they are trapped by the horizontal magnetic field. The ratio of CR to kinetic energies saturates as soon as the vertical magnetic-field component becomes significant, due to buoyancy, enabling the diffusive transport of CRs out of the disk.
When the cosmic-ray diffusion-coefficients are higher, one can find that the ratio of $\mathrm{CR}$ to kinetic energies is lower. We note that cosmic rays find an easier way of leaving the disk when the parallel and perpendicular diffusion coefficients are higher. Shown in Fig. 11, the results indicate that the ratio of CR to kinetic energies anticorrelates with both: the parallel and perpendicular CR diffusion-coefficients. Due to the aforementioned timestep limitation, in the simulations presented in this paper we could only adopt the values of $K_{\|}$reaching at most $3 \times 10^{28} \mathrm{~cm}^{2} \mathrm{~s}^{-1}$, which are still lower than the value of $10^{29} \mathrm{~cm}^{2} \mathrm{~s}^{-1}$ mentioned by other authors (e.g. Jokipii 1999). Although the lowering the CR energy with the magnitude of the parallel CR diffusion coefficient appears a promising solution, one should not expect values as high as $K_{\|} \simeq 10^{29} \mathrm{~cm}^{2} \mathrm{~s}^{-1}$ to reduce the problem of the $\mathrm{CR}$ energy excess. 


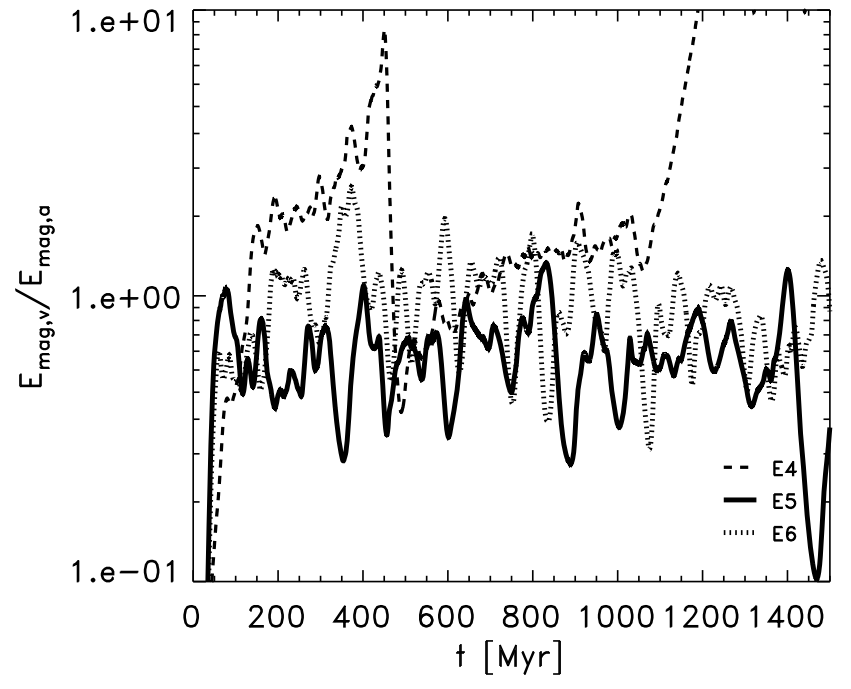

Fig. 10. Time evolution of the ratio of energies of vertical to horizontal magnetic field components for different values of the perpendicular CR diffusion coefficients $K_{\|}=3 \times 10^{4}$ and $K_{\perp}=1 \times 10^{4}$ (E4), $K_{\|}=1 \times 10^{5}$ and $K_{\perp}=1 \times 10^{3}(\mathrm{E} 5)$ and $K_{\|}=1 \times 10^{5}, K_{\perp}=3 \times 10^{3}$ (E6). All diffusion coefficients Gaven in units $\mathrm{pc}^{2} \mathrm{Myr}^{-1}$.

Another factor, that may significantly influence the relationship between CR and other form of energies, is the choice of periodic and shear-periodic boundary conditions in the horizontal directions of the computational box. In true galactic disks, $\mathrm{CR}$ diffusion is expected to occur along the horizontal magnetic field lines. In the case of periodic-type boundary conditions, CRs are trapped inside the disk volume by a predominantly horizontal magnetic-field. This type of trapping can be released only in the global galactic-disk simulations.

\section{Summary and conclusions}

We have described an extensive series of simulations and presented a parameter study of the CR-driven dynamo in a galaxy, characterized by parameters that are typical of the Milky Way galaxy at galactocentric radius of $R_{\mathrm{G}}=5 \mathrm{kpc}$. We considered the magnetic diffusivity, as well as the parallel and perpendicular CR diffusion coefficients to be free parameters, and dedicated simulation series have been performed to investigate their influence on the efficiency of the CR-driven dynamo process. The results of the parameter study can be summarized as follows:

(1) The magnitude of the magnetic diffusivity influences the efficiency of the magnetic-field amplification. The most favorable value of magnetic diffusivity is $100 \mathrm{pc}^{2} \mathrm{Myr}^{-1} \simeq$ $3 \times 10^{25} \mathrm{~cm}^{2} \mathrm{~s}^{-1}$, value comparable to, although lower than, the value of the turbulent diffusivity of the ISM deduced from observational data.

(2) The efficiency of the magnetic-field amplification is enhanced by the temporal modulation of the CR supply. An effect of this kind may be associated with the periodicity of the star-formation and supernova activity induced by the spiral arms. The enhancement is apparent at lower values of magnetic diffusivity and is less significant at the optimal value of $\eta=100 \mathrm{pc}^{2} \mathrm{Myr}^{-1}$.

(3) The magnetic-field amplification rate is relatively insensitive to the magnitude of the $\mathrm{SN}$-rate for $\mathrm{SN}$ rates spanning one

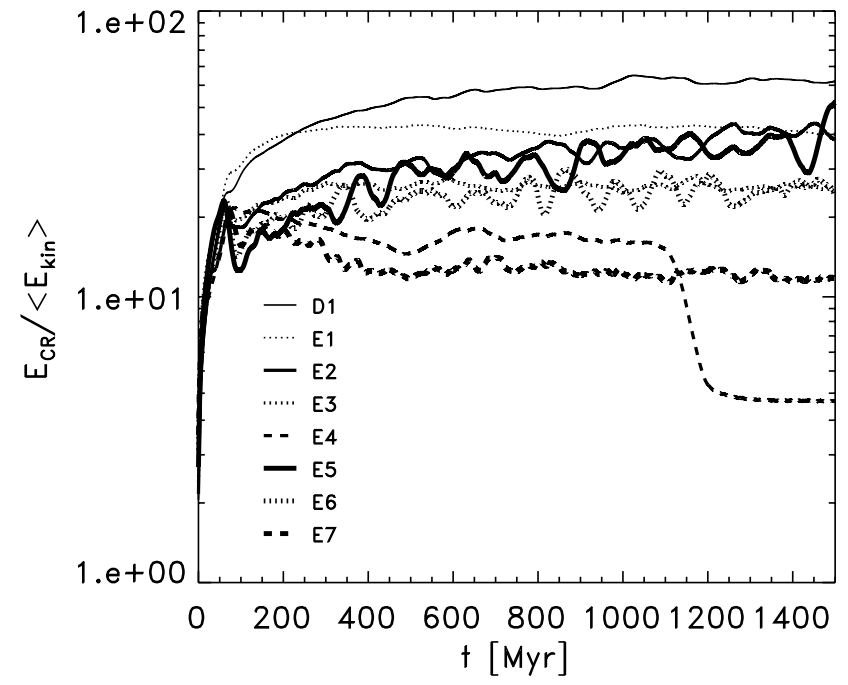

Fig. 11. Time evolution in the ratio of CR to time-averaged kinetic energy for different values of the CR diffusion coefficients in simulation series E. Line assignments are the same as in Fig. 9.

decade below the value $f_{\mathrm{SN}}=130 \mathrm{kpc}^{-2} \mathrm{Myr}^{-1}$, which are typical for the galactocentric radius $R_{\mathrm{G}}=5 \mathrm{kpc}$. We note also that magnetic-field amplification saturates at the level of equipartition in the magnetic and kinetic energies at all supernova rates for which amplification holds. The magnetic field is no longer amplified, if the SN rate is enhanced further by factors of 2 and 4 with respect to realistic values, while other quantities (such as e.g. gas column density) remain fixed.

(4) Magnetic-field amplification in the CR-driven dynamo relies on the anisotropic diffusion of cosmic rays. From the limited set of simulations of series E, one can deduce that the magnetic-field amplification is possible only for $K_{\perp} \leq$ $3 \times 10^{3} \mathrm{pc}^{2} \mathrm{Myr}^{-1} \simeq 10^{27} \mathrm{~cm}^{2} \mathrm{~s}^{-1}$, and for all considered values of the parallel diffusion-coefficient $K_{\|}$in the range $3-30 \times 10^{27} \mathrm{~cm}^{2} \mathrm{~s}^{-1}$. Therefore, the $5 \%$ ratio of the perpendicular to parallel diffusion coefficients postulated by Giacalone \& Jokipii (1999) falls within the amplification range.

(5) By varying the parameters of magnetic diffusivity, supernova rate, and the CR diffusion coefficients, we have found that the favorable conditions for magnetic-field amplification correspond to approximately equal energies of the vertical and azimuthal magnetic field components in the case of the buoyancy-driven dynamo. An excess or deficit of the vertical magnetic field with respect to the azimuthal one corresponds to a significantly less efficient amplification or even a decay in the magnetic field.

(6) We recall problem indicated previously by Snodin et al. (2006), that in all simulations the CR energy in the computational domain exceeds the turbulent kinetic energy and magnetic energy by more than one order of magnitudes. The lowest ratios of CR to kinetic energies also correspond to the highest values of the parallel diffusion coefficient. It seems implausible, however, that an increase in the diffusion coefficients to fully realistic values would reduce the excess of cosmic-ray energy in the disk. It also seems that the ratio of CR to other forms of energy in the ISM is not yet well constrained on observational grounds (Strong et al. 2007). 
On the other hand, the currently used shearing-box approximation does not permit CRs to leave the disk by means of diffusion along the predominantly horizontal magnetic field. Therefore, we suggest that future studies of the CR-driven dynamo, designed to solve this problem, should be completed in the framework of global galactic-disk simulations.

Acknowledgements. This work was supported from the Polish Committee for Scientific Research (KBN) through the grants PB 0656/P03D/2004/26 and 2693/H03/2006/31.

\section{References}

Beck, R., \& Krause, M. 2005, Astron. Nachr., 326, 414

Berezinskii, V. S., Bulanov, S. V., Dogiel, V. A., \& Ptuskin, V. S. 1990, Astrophysics of cosmic rays, ed. V. L. Ginzburg (Amsterdam: North-Holland) Chi, X., \& Wolfendale, A. W. 1993, Nature, 362, 610 Ferriere, K. 1998, ApJ, 497, 759

Fitt, A. J., \& Alexander, P. 1993, MNRAS, 261, 445

Giacalone, J., \& Jokipii, J. R. 1999, ApJ, 520, 204

Gressel, O., Elstner, D., Ziegler, U., \& Rüdiger, G. 2008a, A\&A, 486, L35

Gressel, O., Ziegler, U., Elstner, D., \& Rüdiger, G. 2008b, Astron. Nachr., 329, 619

Hanasz, M., \& Lesch, H. 1993, A\&A, 278, 561

Hanasz, M., \& Lesch, H. 1997, A\&A, 321, 1007

Hanasz, M., \& Lesch, H. 1998, A\&A, 332, 77

Hanasz, M., \& Lesch, H. 2000, ApJ, 543, 235
Hanasz, M., \& Lesch, H. 2001, Space Sci. Rev., 99, 231

Hanasz, M., \& Lesch, H. 2003a, A\&A, 404, 389

Hanasz, M., \& Lesch, H. 2003b, A\&A, 412, 331

Hanasz, M., Otmianowska-Mazur, K., \& Lesch, H. 2002, A\&A, 386, 347

Hanasz, M., Kowal, G., Otmianowska-Mazur, K., \& Lesch, H. 2004, ApJ, 605, L33

Hanasz, M., Otmianowska-Mazur, K., Kowal, G., \& Lesch, H. 2006, Astron. Nachr., 327, 469

Hawley, J. F., Gammie, C. F., \& Balbus, S. A. 1995, ApJ, 440, 742

Heesen, V., Krause, M., Beck, R., \& Dettmar, R.-J. 2007, Astron. Nachr., 328, 637

Heesen, V., Beck, R., Krause, M., \& Dettmar, R.-J. 2009, A\&A, 494, 563

Jokipii, R. 1999, in Interstellar Turbulence, ed. J. Franco, \& A. Carraminana, 70

Kowal, G., Hanasz, M., \& Otmianowska-Mazur, K. 2003, A\&A, 404, 533

Kowal, G., Otmianowska-Mazur, K., \& Hanasz, M. 2006, A\&A, 445, 915

Lesch, H., \& Hanasz, M. 2003, A\&A, 401, 809

Maron, J., \& Blackman, E. G. 2002, ApJ, 566, L41

Maron, J., Cowley, S., \& McWilliams, J. 2004, ApJ, 603, 569

Otmianowska-Mazur, K. 2003, A\&A, 408, 817

Otmianowska-Mazur, K., Kowal, G., \& Hanasz, M. 2007, ApJ, 668, 110

Parker, E. N. 1992, ApJ, 401, 137

Ryu, D., Kim, J., Hong, S. S., \& Jones, T. W. 2003, ApJ, 589, 338

Schlickeiser, R., \& Lerche, I. 1985, A\&A, 151, 151

Snodin, A. P., Brandenburg, A., Mee, A. J., \& Shukurov, A. 2006, MNRAS, 373 , 643

Stone, J. M., \& Norman, M. L. 1992a, ApJS, 80, 753

Stone, J. M., \& Norman, M. L. 1992b, ApJS, 80, 791

Strong, A. W., Moskalenko, I. V., \& Ptuskin, V. S. 2007, Ann. Rev. Nucl. Part. Sci., 57, 285

Tanuma, S., Yokoyama, T., Kudoh, T., \& Shibata, K. 2003, ApJ, 582, 215

Vallee, J. P. 1995, A\&A, 296, 819 